\title{
28 Research Square \\ Efficient Homogeneous TEMPO-mediated Oxidation of Cellulose in Lithium Bromide Hydrates
}

\section{Xiaotong Fu}

Qilu university of technology

Fulong Zhang

South China University of Technology

Cuihua Dong

Qilu university of technology

Wenyuan Zhu

Nanjing Forestry University

Kaifeng Xiong

Qilu University of Technology

zhiqiang pang ( $\sim$ pzhq@qlu.edu.cn )

Qilu University of Technology(Shandong Academy of Sciences) https://orcid.org/0000-0002-01090656

\section{Research Article}

Keywords: homogeneous oxidation, TEMPO, lithium bromide hydrates, cellulose

Posted Date: February 12th, 2021

DOI: https://doi.org/10.21203/rs.3.rs-183865/v1

License: (c) (i) This work is licensed under a Creative Commons Attribution 4.0 International License.

Read Full License

Version of Record: A version of this preprint was published at International Journal of Biological Macromolecules on November 1st, 2021. See the published version at https://doi.org/10.1016/j.jbiomac.2021.09.104. 


\section{Abstract}

An appropriate cellulose-dissolving solvent is critical for the homogeneous oxidation of cellulose using TEMPO (2, 2, 6, 6-tetramethylpiperidine-1-oxyl)-mediated system. Herein, TEMPO/ $\mathrm{NaClO} / \mathrm{NaClO}_{2}$ system in lithium bromide hydrates (LBHs) was developed for the homogeneous selective-oxidation of cellulose, which was two-stage protocol involving cellulose dissolution and homogeneous oxidation. Specifically, cellulose was firstly dissolved in $\mathrm{LiBr} \cdot 3.5 \mathrm{H}_{2} \mathrm{O}$ and offered the optimal $\mathrm{pH}$ (5.6) for the subsequent $\mathrm{TEMPO} / \mathrm{NaClO} / \mathrm{NaClO}_{2}$ oxidation without precipitation of the cellulose chains. Effect of reaction conditions on cellulose oxidation was investigated. The results showed that high degree of oxidation (DO) and evenly distributed carboxyl could be achieved. The particle size gradually decreased with DO, and oxidized cellulose with high water solubility and amorphous structure could be obtained.

Furthermore, FT-IR, ${ }^{13} \mathrm{C}$ NMR and fractionation analysis verified that cellulose was successfully converted and the carboxyl uniformly distributed onto the cellulose chains. This TEMPO-mediated system using LBHs as solvent presented an efficient method on the homogeneous selective oxidation of primary hydroxyl in cellulose.

\section{Highlights}

- $\mathrm{LiBr} \times 5 \mathrm{H}_{2} \mathrm{O}$ was suitable solvent for homogeneous oxidation of cellulose.

- Dissolved cellulose was selectively oxidized by TEMPO/ $\mathrm{NaClO} / \mathrm{NaClO}_{2}$.

- Cellulose at high DO features water solubility and amorphous structure.

- Dissolved cellulose with different DP could be homogenously oxidized.

\section{Introduction}

Cellulose is the most abundant renewable resource synthesized in the biosphere (Chandrakant \& Bisaria, 1998, Dai et al. 2020). With dwindling fossil petroleum reserves and global environmental deterioration, cellulose utilization is rapidly flourishing and receiving an extensive attention, both in academic and industrial communities (Lindman, Karlström, \& Stigsson, 2010). Indeed, a pyramid of functional materials have been produced in form of cellulose and its derivatives, such as cellulose nanocrystal (CNC), dissolving pulp, carboxymethyl cellulose and nitrocellulose (Fu et al., 2020; Peng, Dhar, Liu, \& Tam, 2011). Particularly, chemical modification could endow novel functionality and property to cellulose via introducing various functional groups, and applications of cellulose could be further expanded (Isik, Sardon, \& Mecerreyes, 2014).

Cellulose is linear homopolymer consisting of $\beta$-1, 4-linked D-anhydroglucopyranose units with three hydroxyls at $\mathrm{C}_{2}, \mathrm{C}_{3}$ and $\mathrm{C}_{6}$ position (Bian et al., 2012). The extensive intra- and intermolecular hydrogenbond network confer the semicrysalline nature of cellulose, and cellulose chains are tightly ordered packed in the crystalline region. Additionally, cellulose is inherently amphiphilic (Medronho \& Lindman, 2014). The equatorial direction of a glucopyranose ring located with all three hydroxyl groups is 
hydrophilic (Hrmova et al., 2002). On the other side, the hydrogen atoms of C-H bond are located at the axial position of the ring, which endows cellulose hydrophobic character (Biermann, Hädicke, Koltzenburg, \& Müller-Plathe, 2001). Therefore, cellulose is insoluble in most common solvents, and chemical modifications of cellulose without suitable solvent are generally heterogeneously performed for the inferior accessibility of hydroxyls (Bialik et al., 2016). In contrast to heterogeneous reaction, homogeneous modification allows for intimate interaction between cellulose chains and modifying agents. Thus, reaction rate could be accelerated, and cellulose derivatives with high degree of substitution and uniformity could be obtained (Heinze, Schwikal, \& Barthel, 2005).

Exploring solvents for efficient cellulose dissolution has never been relenting. Many derivatizing and nonderivatizing cellulose solvents have been discovered, such as copper ethylene diamine solution, $N, N-$ dimethyl acetamide/lithium chloride (DMAc/LiCl), dimethyl sulfoxide/tetrabutylammonium fluoride (DMSO/TBAF), ionic liquids (ILs), deep eutectic solvents (DES), lithium perchlorate trihydrate $\left(\mathrm{LiClO}_{4} \cdot 3 \mathrm{H}_{2} \mathrm{O}\right)$, and lithium thiocyanate hydrate $\left(\mathrm{LiSCN} \cdot 2 \mathrm{H}_{2} \mathrm{O}\right)($ Heinze \& Koschella, 2005; Wang, Lu, \& Zhang, 2016). However, solvents and conditions for cellulose dissolution are inappropriate for the subsequent chemical modification in most cases, and many homogeneous modification systems of cellulose has not been fabricated, for example, 2, 2, 6, 6-tetramethylpiperidine-1-oxyl radical (TEMPO)mediated oxidation of cellulose.

TEMPO and its derivatives are water-soluble, commercially available and stable nitroxyl radicals, which opened a new field of efficient and selective oxidation of hydroxyls under mild aqueous conditions (Isogai, Saito, \& Fukuzumi, 2011). The primary hydroxyls in polysaccharides could be selectively converted into carboxylate groups, where catalytic amounts of TEMPO and $\mathrm{NaBr}$ were dissolved in polysaccharide solutions at $\mathrm{pH} 10-11$ using $\mathrm{NaClO}$ as a primary oxidant (DiFlavio et al., 2006). However, TEMPO-mediated oxidation of cellulose has been primarily performed as liquid-solid heterogeneous reactions, where the reactivity of cellulose substantially differed within the crystalline and amorphous zones. Specifically, oxidation primarily occurred in the amorphous zone, and the crystalline zone of cellulose cannot be effectively oxidized due to its inaccessibility. The TEMPO-mediated oxidation of the natural cellulose cannot synchronously proceed among the crystalline and amorphous zones. Thus, the controllability of oxidation is inferior with extremely low degree of oxidation (DO) for the crystalline zone, and the oxidative groups are mainly distributed on the amorphous region and surface of crystalline cellulose (Isogai, Hänninen, Fujisawa, \& Saito, 2018). Generally, this preferential oxidation of the amorphous cellulose following with the mild mechanical disintegration treatment was used to prepare nanofibers, which serve as an additive in food, coating, daily necessities, cosmetics and other products (Okita, Fujisawa, Saito, \& Isogai, 2011).

So far, TEMPO-mediated system for cellulose oxidation mainly includes TEMPO/ $\mathrm{NaBr} / \mathrm{NaClO}$ and TEMPO/ $\mathrm{NaClO} / \mathrm{NaClO}_{2}$ system (Isogai, Hänninen, Fujisawa, \& Saito, 2018). Cellulose could be effectively oxidized using TEMPO/NaBr/NaClO system in a relatively short time (Saito \& Isogai, 2004). However, pH of reaction should be strictly controlled at ca. $10 \pm 0.5$ and cellulose is readily degraded into oligosaccharides or monosaccharides at alkaline condition (Hao et al., 2017; T. Isogai, Yanagisawa, \& A. 
Isogai, 2008). Comparatively, TEMPO/ $\mathrm{NaClO} / \mathrm{NaClO}_{2}$ system was performed at weak acidic condition, and degradation of cellulose was significantly reduced by avoiding alkaline $\beta$-elimination reaction (Saito, Hirota, Tamura, \& Isogai, 2010). In addition, reactivity of cellulose can be greatly enhanced by decrystallization of the natural cellulose. Therefore, cellulose $\nabla$ and cellulose $\otimes$ were more readily to be oxidized compared to the natural cellulose (Da Silva Perez, Montanari, \& Vignon, 2003; Tang et al., 2017), however, the homogeneous oxidation still cannot be achieved with low uniformity of the final products. Summarily, reports concerning TEMPO-mediated oxidation of cellulose was concentrated on heterogeneous conditions, and homogeneous reaction has not been reported hitherto. The reactivity of $\mathrm{C}_{6}-\mathrm{OH}$ in crystalline zone were severely inhibited in the traditional heterogenous system due to compact $\mathrm{H}$-bond network, however, the cellulose chains in the crystalline zone could be individually dispersed using the homogenous system. Thus, the accessibility and reactivity of $\mathrm{C}_{6}-\mathrm{OH}$ both in crystalline and amorphous zone could be homogenized and boosted. To enhance reactivity and uniformity of cellulose, developing an efficient homogeneous TEMPO-mediated oxidation of cellulose is pressing, and exploring an efficient and suitable solvent is critical.

Presently, cellulose could be readily dissolved in lithium bromide hydrates (LBHs) (Yoo, Zhang, \& Pan, 2017; Yang et al. 2014). LBHs is an aqueous solvent for cellulose, and cellulose/LBHs solution itself is at weak acidity ( $\mathrm{pH} 3-5)$. Additionally, the cellulose/LBHs solution was stable enough for chemical modification. Therefore, $\mathrm{LBHs}$ as solvent is an ideal candidate for homogeneous oxidation of cellulose using TEMPO/ $\mathrm{NaClO} / \mathrm{NaClO}_{2}$ system. Here, we firstly dissolved cellulose in $\mathrm{LBHs}$ at $110^{\circ} \mathrm{C}$, then the homogeneous TEMPO/ $\mathrm{NaClO} / \mathrm{NaClO}_{2}$ reaction was performed in the cellulose/LBHs solution without further adjusting the $\mathrm{pH}$. The oxidized cellulose with high uniformity could be prepared using this homogeneous TEMPO-mediated system. In addition, the uniformly distributed oxidized groups endow many novel potential applications of cellulose. For example, the homogeneous oxidized cellulose could be used for as a hemostatic for moderate bleeding which cannot be sutured or ligated in surgery.

\section{Experimental \\ 2.1. Materials}

Microcrystalline cellulose (MCC) with degree of polymerization (DP) of 190 (MCC-190) and 660 (MCC660), and dissolving pulp with DP of 610 (DvP-610) and 900 (Dvp-900) were provided by Anhui Shanhe Pharmaceutical Co. All other chemicals, including TEMPO, 4-methoxy-TEMPO, 4-acetamido-TEMPO, $\mathrm{NaClO}, \mathrm{NaClO}_{2}$, and $\mathrm{LiBr}$ were purchased from Aladdin Industrial Corporation. Dialysis bags with cut-off molecular weight of 200D were purchased from Ruida Ever-bright Ltd. Co.

\subsection{Preparation of TEMPO-Oxidized Cellulose}

MCC or dissolving pulp $(1.0 \mathrm{~g})$ was completely dissolved in $\mathrm{LiBr} \cdot 3.5 \mathrm{H}_{2} \mathrm{O}(250 \mathrm{~g})$ at $110{ }^{\circ} \mathrm{C}$ for $20 \mathrm{~min}$ under vigorous magnetic stirring $(800 \mathrm{rpm})$, and homogeneous cellulose solution at concentration of 0.4 $w t \%$ was formed. Then the cellulose solution was cooled to a targeted temperature. Prior to oxidation, 
TEMPO or TEMPO derivatives catalyst $(0.065 \mathrm{mmol})$ and $\mathrm{NaClO}_{2}(7.5 \mathrm{mmol})$ were added into the solution and mixed for $5 \mathrm{~min}$, then $\mathrm{NaClO}$ solution $(0.5 \mathrm{mmol})$ was dropwise added to initiate the oxidation reaction. After reaction for specified time, the oxidation was quenched by adding an excess of $95 \%$ ethanol solution. The samples were purified by dialyzing against water for $60 \mathrm{~h}$, and the final solutions were freeze-dried to obtain the oxidized celluloses.

The viscosity of LBHs and cellulose solution were measured using a Brookfield DVSRV rational viscometer (Berwyn, Pennsylvania, USA), and temperature of the samples was adjusted by a water bath. The cellulose solution was observed using a PM6000 polarized light microscopy (Jiangnan, Nanjing, China) to monitor recrystallization of cellulose chains. The particle size of MCC and oxidized celluloses were analyzed by a Zetasizer Nano ZS instrument (Malvern Instruments Ltd., Malvern, UK) based on dynamic light scattering. All the sample suspensions were ultrasonically treated (VWR, $45 \mathrm{kHz}$ and 180 W) for 30 min before analysis. For each sample, the measurement was conducted in triplicate. The average data were reported after repeated analyses.

\subsection{Determination of degree of oxidation (DO) of the oxidized celluloses}

DO of the oxidized celluloses was defined as the ratio between the molar amount of the oxidized hydroxymethyl groups and the total anhydroglucose units in the sample, which was determined by the conductimetric titration. Specifically, the cellulose samples (30-50 mg) were suspended in $15 \mathrm{~mL} 0.01 \mathrm{M}$ $\mathrm{HCl}$ solution. After stirring for $10 \mathrm{~min}$ at speed of $500 \mathrm{rpm}$, the suspensions were titrated with $0.1 \mathrm{M} \mathrm{NaOH}$ solution. The conductivity was monitored using a conductivity meter throughout the titration process. DO of the oxidized celluloses was calculated using Eq. (1).

$$
\operatorname{DO}(\%)=\left[162 \times c \times\left(V_{2}-V_{1}\right)\right] /\left[w-36 \times c \times\left(V_{2}-V_{1}\right)\right] \times 100
$$

where $c$ is concentration of $\mathrm{NaOH}$ solution, $\mathrm{M} ; \mathrm{NaOH}$ volume at the end of the first-stage linear change of conductivity is $V_{1}$ and $\mathrm{NaOH}$ volume at the start of the second-stage linear change of conductivity is $V_{2}$, $\mathrm{mL} ; w$ is the oven-dried weight of the oxidized sample, g; 162 is the molecular weight of anhydroglucopyranose unit in cellulose chain; 36 corresponds to the molecular weight difference between an oxidized group in sodium salt form and the $\mathrm{C}_{6}$ primary hydroxyl structure.

\subsection{Determination of Degree of Polymerization (DP) of the oxidized celluloses}

The oxidized celluloses $(0.25 \mathrm{~g})$ were dissolved in the cupriethylenediamine solution ( $50 \%)$. The viscosity of the sample is measured by the time that the solution flows through the viscosity tube at $25^{\circ} \mathrm{C}$, and the triplicate average value is calculated. The viscosimetric average DP of the oxidized celluloses was 
calculated from the instrinsic viscosities $([\eta])$ of the corresponding solution at $25^{\circ} \mathrm{C}$, using the relation $\mathrm{DP}^{0.905}=0.75[\eta]$.

\subsection{Fourier transform infrared spectroscopy (FT-IR) analysis}

The functional groups in the oxidized celluloses and MCC were analyzed using an alpha FT-IR (Bruker, Karlsruhe, Germany) by $\mathrm{KBr}$ pellet sampling technique. In order to convert - $\mathrm{COONa}$ into $-\mathrm{COOH}$ groups, the oxidized products were acidified with a dilute $\mathrm{HCl}$ at $\mathrm{pH} 2$ for $15 \mathrm{~min}$. Then the solutions were purified by dialyzing against water for $60 \mathrm{~h}$, and the final solutions were freeze-dried to obtain the acidified oxidized celluloses. The MCC and oxidized celluloses were ground and mixed with $\mathrm{KBr}$ and pressed in an evacuated die under proper pressure. The spectra were collected for 16 scans within wavenumber ranged from 500 to $4000 \mathrm{~cm}^{-1}$ at $2-\mathrm{cm}^{-1}$ resolution.

\subsection{Thermal properties of MCC and the oxidized celluloses}

The thermal properties of MCC and the oxidized celluloses were analyzed using a Q50 thermogravimetric analysis (TGA) system (TA-Instruments, New Castle, USA). The measurement was carried out under a flowing nitrogen atmosphere $(20 \mathrm{~mL} / \mathrm{min})$, and the samples were treated from $50{ }^{\circ} \mathrm{C}$ to $650{ }^{\circ} \mathrm{C}$ at heating rate of $10^{\circ} \mathrm{C} / \mathrm{min}$.

\subsection{X-ray diffraction (XRD) analysis}

The crystalline structure of MCC and the oxidized celluloses were analyzed by XRD technique. Prior to analysis, the samples were pressed into flattened sheets on a sample holder, then the samples were scanned on a D8 Advance X-ray diffractometer (Bruker, Karlsruhe, Germany), The spectra were collected with a Co radiation source at $40 \mathrm{~kW}$ and $35 \mathrm{~mA}$, over a $2 \theta$ range from $5^{\circ}$ to $50^{\circ}$ at a scan speed of 0.18 $\%$ min, and a step size of $0.018^{\circ}$. And the crystallinity index (Crl) of cellulose was calculated according to method of (Segal et al. 1959).

\subsection{Fractionation of the oxidized celluloses}

The oxidized celluloses were fractionated into soluble and insoluble portions. Firstly, the oxidized cellulose $(100 \mathrm{mg})$ were mixed with $\mathrm{H}_{2} \mathrm{O}$ /acetone solution (1:2) at room temperature for 20 min. Then the solution was filtered using a T-50.1L liquid-phase filtration system (Jinteng, Tianjin, China) with organic microfiltration membrane $(0.2 \mu \mathrm{m})$. The insoluble portion was obtained by freeze-drying of the precipitate cake, and the soluble portion was obtained by freeze-drying of the filtrate. Finally, DO of the fractionated samples were determined accordingly method in Sect. 2.3.

\subsection{The solid-state ${ }^{13} \mathrm{C}$ nuclear magnetic resonance (NMR) analysis}

The solid-state ${ }^{13} \mathrm{C}$ NMR spectra of MCC and the oxidized cellulose were obtained using AVANCE $\triangle \mathrm{HD}$ 600 superconducting Fourier transform NMR spectrometry (Bruker, Karlsruhe, Germany) equipped with a radio frequency (RF) generator. Specifically, freeze-dried samples (200 mg) were tested at $303.0 \mathrm{~K}$. The 
detailed collecting and processing parameters were number of scans of 2000, receiver gain of 567, acquisition time of $0.0344 \mathrm{~s}$, relaxation delay of $1.5 \mathrm{~s}$, pulse width of $4.55 \mathrm{~s}$, spectra frequency at $100.61 \mathrm{~Hz}$, and spectra width of $29761.9 \mathrm{~Hz}$. And the spectra were analyzed using Bruker TopSpin 3.0.

\subsection{The molecular weight and distribution analysis of the oxidized celluloses}

The molecular weight and distribution of the oxidized celluloses were measured using size-exclusion chromatography (SEC) furnished with a refractive index detector (RID), and $0.1 \mathrm{M} \mathrm{NaCl}$ solution was used as eluent. The oxidized product (around $0.1 \mathrm{mg}$ ) was dissolved in $0.1 \mathrm{M} \mathrm{NaCl}$ solution and the solution was filtered with a $0.20 \mu \mathrm{m}$ polytetrafluoroethylene (PTFE) membrane (Milex-LG, Milipore, USA). An $8 \times$ $300 \mathrm{~mm}$ (inner diameter $\times$ length) Shodex SB-806MHQ column with polyhydroxy methacrylate gel as packing was used, and poly ethylene glycol with DP of 200, 400, and 600 were used as standard for calibration. Data acquisition and processing was performed in LCsolution GPC software.

\section{Results And Discussion}

\subsection{Cellulose dissolution and properties of cellulose solution}

The natural cellulose could be readily dissolved in LBHs, and cellulose chains were homogeneous dispersed with high accessibility to reagents. In our previous study, the optimal temperature for MCC dissolution is $110^{\circ} \mathrm{C}$, and the crystalline region could be thoroughly deconstructed for ca. 20 min. However, the temperature for cellulose dissolution and TEMPO-mediated oxidation was not concerted, and TEMPO-mediated oxidation was typically performed at relatively low temperature. Thus, the properties of cellulose solution were compared with varying cellulose concentration and temperature (Fig. 1). The viscosity of LBHs was low and negligibly affected by temperature, and viscosity of $\mathrm{LiBr} \cdot 3.5 \mathrm{H}_{2} \mathrm{O}$ only increased from $7.5 \mathrm{~Pa} \cdot \mathrm{s}$ at $110{ }^{\circ} \mathrm{C}$ to $16.9 \mathrm{~Pa} \cdot \mathrm{s} 20^{\circ} \mathrm{C}$. However, viscosity significantly increased for cellulose dissolution, for example, viscosity of $0.6 \mathrm{wt} \%$ cellulose and $0.4 \mathrm{wt} \%$ cellulose in $\mathrm{LiBr} \cdot 3.5 \mathrm{H}_{2} \mathrm{O}$ at $110^{\circ} \mathrm{C}$ are $1240 \mathrm{~Pa} \cdot \mathrm{s}$ and $680 \mathrm{~Pa} \cdot \mathrm{s}$, respectively. Undoubtedly, the long cellulose chains substantially altered the rheological properties, and high cellulose concentration resulted to high viscosity and inferior mass transfer. Except cellulose concentration, viscosity also was significantly affected by temperature (Lasseuguette et al. 2008). The mobility of cellulose chains became weak at low temperature, and viscosity increased sharply with decreasing temperate. For example, the viscosity (680 $\mathrm{Pa} \cdot \mathrm{s}$ ) of $0.4 \mathrm{wt} \%$ cellulose solution at $110^{\circ} \mathrm{C}$ considerably increased to $1130 \mathrm{~Pa} \cdot \mathrm{s}$ at $40^{\circ} \mathrm{C}$ and $1470 \mathrm{~Pa} \cdot \mathrm{s}$ at $20^{\circ} \mathrm{C}$. Thus, the mixing and mass transfer during oxidation reaction could be troublesome, which retarded oxidation of cellulose. Considering increasing viscosity of solution at low temperature during oxidation stage, cellulose concentration of $0.4 \mathrm{wt} \%$ was used for homogeneous TEMPO-mediated oxidation. In addition, the $\mathrm{pH}(5.6)$ of $\mathrm{LiBr} \cdot 3.5 \mathrm{H}_{2} \mathrm{O}$ was suitable for the subsequent TEMPO/ $\mathrm{NaClO} / \mathrm{NaClO}_{2}$ oxidation, and adjusting $\mathrm{pH}$ could be avoided. 


\subsection{Effect of reaction conditions on oxidation of cellulose}

Effect of reaction conditions on oxidation of cellulose using TEMPO $/ \mathrm{NaClO} / \mathrm{NaClO}_{2}$ system was investigated. Firstly, effect of reaction time on oxidation of cellulose was conducted $25^{\circ} \mathrm{C}$ in $\mathrm{LiBr} \cdot 3 \cdot 5 \mathrm{H}_{2} \mathrm{O}$ (Fig. 2A). Apparently, DO of the oxidized products was closely related to reaction time. The reaction was monitored during cellulose oxidation. Specifically, a droplet of solution was withdrawn at interval of 60 min and observed using a polarized-light spectroscopy, and no aggregates of the crystalline cellulose were detected and the homogenous oxidation was guaranteed. Owing to the homogenous reaction and high accessibility of substrate, DO of the products increased rapidly at the initial stage and reached to $22.7 \%$ for $24 \mathrm{~h}$. DO of products could be further enhanced by increasing reaction time, for example, DO of the products increased to $33.2 \%$ when reaction time was prolonged to $96 \mathrm{~h}$. Moreover, the particle size and appearance of the final dialyzed products were recorded. The particle size of the oxidized products became smaller with reaction time. When DO of product reached $33.2 \%$, the oxidized cellulose could be completely dissolved in water and form transparent solution of oxidized cellulose. Indeed, the introduced carboxylate group are more hydrophilic and solubility of oxidized cellulose could be enhanced. The watersoluble percentage of oxidized celluloses gradually increased with DO, and high content of oxidized groups boosted solubility of oxidized celluloses. In addition, DP of oxidized celluloses decreased slightly with reaction time, and drastic degradation of cellulose could be avoided in this reaction. Thus, watersoluble percentage of final products was primarily due to introduction of the oxidized groups. The solution of final products was also examined by polarized-light spectroscopy. The crystalline cellulose became smaller and loose for long reaction time, and the oxidative groups also hindered the regeneration of the crystalline cellulose. Correspondingly, the uniform solution was achieved for reaction time of $96 \mathrm{~h}$.

Effect of temperature on cellulose oxidation was investigated in $\mathrm{LiBr} \cdot 3.5 \mathrm{H}_{2} \mathrm{O}$ for reaction time of $72 \mathrm{~h}$ (Fig. 2B). Essentially, both the oxidation and side reactions rate could be kinetically accelerated at high temperature. However, the maximal DO (63.8\%) was achieved at $40^{\circ} \mathrm{C}$, and DO of final products diminished with further growing temperature. Conceivably, the severely degradation of cellulose at high temperature simultaneously occurred, and the oxidized cellulose at DP low may lost during dialysis. Thus, high temperature resulted to the low efficiency of oxidization, and the oxidized product at high temperature had low DO and DP. Water-soluble percentage of the oxidized celluloses closely correlated with $\mathrm{DO}$, and the product at $40^{\circ} \mathrm{C}$ has the highest water-soluble percentage. Moreover, the crystalline aggregates could not be observed using polarized-light spectroscopy for the samples treated at high temperature due to high DO.

The catalytic performance with different TEMPO-based structure (TEMPO, 4-methoxy-TEMPO, and 4acetamide-TEMPO) was compared in this homogeneous system at $40{ }^{\circ} \mathrm{C}$ for $72 \mathrm{~h}$ in $\mathrm{LiBr} \cdot 3.5 \mathrm{H}_{2} \mathrm{O}$ (Fig. 2C). All the examined catalysts were efficient in selective oxidization of cellulose. Specifically, DO of the final product using TEMPO (63.84\%) was slightly higher than that of 4-methoxy-TEMPO $(55.70 \%)$ and 4-acetamide-TEMPO (59.00\%). The side groups (methoxy and acetamide) on TEMPO ring may reduce the affinity of catalyst to the substrate via steric effect. Correspondingly, the degradation of cellulose was 
slightly restricted, the oxidized celluloses using substituted TEMPO had higher DP, however, water-soluble percentage of the samples using 4-methoxy-TEMPO and 4-acetamide-TEMPO were low due to its low DO. In addition, both $M_{n}$ and $M_{w}$ of the oxidized celluloses were determined, which slightly decreased with DO, and polydispersity increased faintly (Fig. 2D). Therefore, oxidation extent had little effect on the molecular weight of the oxidized celluloses, and the degradation of cellulose at the optimal conditions could be effectively restricted. In addition, effect of cellulose species with varying DP on the homogeneous oxidation was compared using TEMPO at $40{ }^{\circ} \mathrm{C}$ for $72 \mathrm{~h}$ in $\mathrm{LiBr} \cdot 3.5 \mathrm{H}_{2} \mathrm{O}$ (Fig. 2E). Comparatively, DO of the final products differed slightly with DP of feedstock. The cellulose chains could be homogeneous dispersed in solution after dissolution, which enabled the similar accessibility of the functional groups on the substrates with varying DP. In other word, the cellulose chains with varying length could be uniformly oxidized regardless its origin, suggesting the successful homogeneous oxidation of primary hydroxyl and even distribution of oxidative groups on cellulose chains. Regardless the similar DO, water-soluble percentage of the oxidized celluloses significantly varied due to different DP, and high DP resulted to low water-soluble percentage of the oxidized celluloses. Moreover, high content of the oxidized groups could be achieved by this method. The highest carboxyl content of oxidized cellulose by this homogeneous system $\left(40^{\circ} \mathrm{C}, 72 \mathrm{~h}\right)$ is $63.8 \%$. Comparatively, the carboxyl content of oxidized cellulose by heterogeneous system were 15.8\% (Saito et al. 2009) and 8.3\% (Saito et al. 2005) in reported studies.

\subsection{FT-IR analysis of the oxidized celluloses}

FTIR analysis of the original cellulose and oxidized celluloses with varying DO was conducted (Fig. 3). A sharp peak at $1429 \mathrm{~cm}^{-1}$ in MCC was assigned to symmetric bending of $\mathrm{CH}_{2}$. Due to oxidation of primary hydroxyl, the intensity of this peak in the oxidized celluloses decreased. MCC was decrystallized and hydrogen-bond network broke after oxidization, thus position of this peak red-shifted to a lower wavenumber without inhibition effect of hydrogen-bond. Moreover, the peaks at $1163 \mathrm{~cm}^{-1}$ (C-O-C symmetric bending), $1111 \mathrm{~cm}^{-1}$ (out-of-plane bending of the skeletal ring), $1058 \mathrm{~cm}^{-1}$ (out-of-plane bending of $\mathrm{C}-\mathrm{OH}$ at $\mathrm{C}_{3}$ ), $1032 \mathrm{~cm}^{-1}$ (out-of-plane bending of $\mathrm{C}-0$ at $\mathrm{C}_{6}$ ) in $\mathrm{MCC}$ were gradually merged into a broad peak with growing DO of the oxidized celluloses (Salmén and Bergström 2009). Peak at 897 $\mathrm{cm}^{-1}$ (C-0-C stretching at the $\beta-1-4$ glycosidic linkage) was well kept after oxidation. Particularly, a new peak at $1730 \mathrm{~cm}^{-1}$ assigned to $\mathrm{C}=0$ stretching vibration was observed in the spectra of the oxidized celluloses (Tang et al. 2017), and this peak appeared as a should peak neighboring the strong bending vibration of the absorbed water (around $1630 \mathrm{~cm}^{-1}$ ) (Pang et al. 2018). A weak shoulder peak at 1233 $\mathrm{cm}^{-1}$ also could be discerned, which was assigned to $\mathrm{C}-\mathrm{O}$ stretching vibration in carboxyl group. In addition, the intensity of this peak increased with DO of the oxidized celluloses. All these changes in FTIR spectra verified the successful introduction of the oxidative groups onto the cellulose chains.

\subsection{Thermal analysis of the oxidized celluloses}

The thermal properties of the oxidized celluloses were closely correlated with its physical structure and functional groups, and the thermostability of the oxidized celluloses with varying DO were analyzed by 
TGA technique (Fig. 4). Compared to MCC, the thermostability of the oxidized celluloses decreased with varying extent. Apparently, the extensive weight loss of MCC occurred at a relative narrow temperature range $\left(315-373^{\circ} \mathrm{C}\right)$, where a large amount of volatile was released (Yang et al. 2007, Muhammad et al. 2010). After homogeneous oxidation of primary hydroxyl, the hydrogen-bond network and compact crystalline cellulose were deconstructed, and loose products with active carboxyl formed.

Correspondingly, the oxidized celluloses were more readily decomposed. Therefore, oxidation of cellulose lowered its decomposition temperature, and the stage of the extensive weight loss occurred at a broader temperature range. For example, the extensive weight loss of the sample with DO of $22.74 \%$ ranged from $219^{\circ} \mathrm{C}$ to $336^{\circ} \mathrm{C}$, and the extensive weight loss of the sample with DO of $33.24 \%$ was further lowered to $200-330^{\circ} \mathrm{C}$.

Moreover, the rate of weight loss as a function of temperature were plotted, and the maximum rate of weight loss could be determined at peak of the contour. Undoubtedly, the maximum rate of weight loss of the oxidized celluloses reduced compared to MCC due to lowered thermostability. In respect of the oxidized celluloses using MCC as feedstock, the maximum rate of weight loss decreased with growing DO. Specifically, the maximum rate of weight loss of MCC is $354^{\circ} \mathrm{C}$. The maximum rate of weight loss of the corresponding oxidized celluloses with DO of $22.74 \%$ and $33.24 \%$ decreased to $314^{\circ} \mathrm{C} 221^{\circ} \mathrm{C}$, respectively. In respect of the oxidized cellulose using DvP-900 as feedstock, DP is significantly higher than that of MCC. Regardless its high DO (65.56\%), the maximum rate of weight loss $\left(238^{\circ} \mathrm{C}\right)$ is still higher than that of the sample of DO (33.24\%) using MCC-170. Except DO of the oxidized celluloses, DP of cellulose also accounted for the maximum rate of weight loss and thermostability of the oxidized cellulose.

\subsection{XRD analysis of MCC and the oxidized cellulose}

The crystalline structures of MCC and the oxidized celluloses were analyzed using the XRD technique (Fig. 5). MCC exhibited two diffraction peaks approximately located at $16^{\circ}$ and $22^{\circ}$, which is characteristic for the crystalline cellulose I structure (Das et al. 2010). After dissolution and the selective homogeneous TEMPO-mediated oxidation in LBHs, the characteristic diffraction peaks of cellulose I could not be observed in the oxidized celluloses, and the XRD profile was closely related with DO of the final products. In respect of the sample at low DO, diffraction peaks $\left(20.3^{\circ}\right)$ of cellulose II could be observed, demonstrating the transformation of crystalline cellulose I and partial formation of the crystalline cellulose II (Nomura et al. 2020). Thus, the crystalline cellulose II still could be formed with small amount of introduction of the oxidative groups. The orientation of cellulose chains was not significantly disturbed and the crystallite could be formed. Correspondingly, Crl of cellulose substantially decreased for oxidation. In contrast to the original MCC (73.9\%), Crl of the oxidized cellulose with DO of $22.74 \%$ sharply dropped to $38.41 \%$. However, the characteristic diffraction peaks of crystalline cellulose II could not be detected for the sample with high DO, and hydrogen-bond pattern for crystalline cellulose II failed to form due to attachment of oxidative groups. Correspondingly, the amorphous patter of XRD spectra was detected for sample with DO of $63.8 \%$, and the cellulose chains were randomly distributed. 


\subsection{Fractionation of the oxidized cellulose and distribution oxidation groups}

The oxidized celluloses were fractionated into soluble and insoluble portion using $\mathrm{H}_{2} \mathrm{O}$ /acetone (1:2) solution. Then DO of the fractionated samples was determined and distribution of the oxidative groups on products were analyzed (Fig. 6). Actually, DO of the fractionated samples negligibly changed compared to its original sample. Comparatively, DP of the insoluble portion was negligibly greater than that of the soluble portion. The relative high DO of the soluble portion with low DP may attribute to its rapid diffusion and easy accessibility to the reactive species. Therefore, the oxidative groups were uniformly distributed on the cellulose chains, which further evidenced the homogeneous TEMPOmediated oxidation of cellulose.

\subsection{NMR analysis of cellulose before and after oxidation}

The solid-state ${ }^{13} \mathrm{C}$-NMR analysis of the original cellulose and oxidized cellulose was conducted to further obtain structural information (Fig. 7). The unit of cellulose is anhydroglucopyranosyl. In respect of MCC, the peak at $\delta_{C} 107$ ppm was assigned to $C_{1}$ atom forming glycosidic bond in the cellulose chain, and the $C_{1}$ in the reducing ends of cellulose appeared at $90-100 \mathrm{ppm}$ with weak resonance signal for its relatively low content. The peak at $\delta_{C} 90 \mathrm{ppm}\left(\mathrm{C}_{4}\right)$ and the broad resonance signal at $\delta_{C} 81-98 \mathrm{ppm}\left(\mathrm{C}_{2}\right.$, $\mathrm{C}_{3}$ and $\mathrm{C}_{5}$ ) were also detected (Isogai and Kato, 1998, Tahiri and Vignon, 2000). These resonance signals could also be observed in the spectra of the oxidized cellulose. During oxidation reaction, the formed nitrosonium ion by TEMPO selectively oxidized the primary hydroxyl into aldehyde at acid or neutral media, then $\mathrm{C}_{6}$-aldehyde group as intermediate was in situ converted into carboxylate groups by $\mathrm{NaClO}_{2}$ (Hirota, Tamura, Saito, \& Isogai, 2009). The $\mathrm{C}_{6}=\mathrm{O}$ is the only carboxyl group after TEMPO/ $\mathrm{NaClO} / \mathrm{NaClO}_{2}$ treatment. Correspondingly, the selective oxidation of primary hydroxyl on $\mathrm{C}_{6}$ resulted to significant change of $\mathrm{C}_{6}$ resonance signal. A single peak $\left(\delta_{C} 67.5 \mathrm{ppm}\right)$ assigned to $\mathrm{C}_{6}-\mathrm{OH}$ in $\mathrm{MCC}$ spectra could be observed. Comparatively, the partial conversion of primary hydroxyl to carboxyl led to splitting of $\mathrm{C}_{6}$ resonance signal. On one hand, the intrinsic resonance signal of $\mathrm{C}_{6}-\mathrm{OH}$ could be detected with lowered intensity. On the other hand, a new resonance signal $\left(\delta_{C} 176 \mathrm{ppm}\right)$ emerged due formation of $C_{6}=0$, and this phenomenon also was also confirmed by (Sang et al., 2017). Except this, no other new peak could be de detected, and the hydroxyls on $\mathrm{C}_{2}$ and $\mathrm{C}_{3}$ were well conserved. Therefore, a vast amount of carboxyl group was attached to the oxidized products, and the primary alcohol groups in the native cellulose were substantially converted. The resonance intensity of $\mathrm{C}_{6}=\mathrm{O}$ signal of the water-soluble product is slightly higher. Correspondingly, more carboxyl groups were introduced onto cellulose chains, which boost the hydrophilic of the oxidized cellulose. In addition, the conversion of primary hydroxyl also could be quantified by integral area of resonance signal from $\mathrm{C}_{6}-\mathrm{OH}$ and $\mathrm{C}_{6}=\mathrm{O}$. The calculated $\mathrm{DO}$ from ${ }^{13} \mathrm{C} \mathrm{NMR}$ spectra (58.5\%) was close to the result by conductimetric titration method (63.8\%). Thus, the selective oxidation of primary hydroxyl could be corroborated. 


\section{Conclusion}

A novel homogeneous TEMPO-mediated oxidation of cellulose was fabricated using LBHs as solvent, where efficient and selective oxidization of primary hydroxy could be achieved. Firstly, cellulose was dissolved in $\mathrm{LiBr} \cdot 3.5 \mathrm{H}_{2} \mathrm{O}$, then the homogeneously dispersed cellulose chains were oxidized by TEMPO/ $\mathrm{NaClO} / \mathrm{NaClO}_{2}$ system. The crystalline of cellulose was deconstructed, and the oxidative groups were evenly distributed on cellulose chains with high DO. The highest carboxyl content of oxidized cellulose which prepared by this homogeneous system at $40^{\circ} \mathrm{C}$ for $72 \mathrm{~h}$ is $3.8 \mathrm{mmol} / \mathrm{g}$. While the carboxyl content of oxidized cellulose which prepared by heterogeneous system is much lower than it. And the highest DO of the oxidized cellulose was $58.5 \%$ by solid-state NMR characterization, which is consistent with the data of conductance titration. Correspondingly, the oxidized products have characteristics of water solubility and amorphous structure. This TEMPO-mediated oxidization in LBHs offers an efficient method for preparation of oxidized cellulose with high uniformity.

\section{Declarations}

\section{Funding}

The authors are grateful for financial support from Natural Science Foundation of Shandong Province (ZR2017MC007), International Cooperation Funding of Qilu University of Technology (QLUTGJHZ2018030, QLUTGJHZ2018027), Jiangsu Provincial Key Laboratory of Pulp and Paper Science and Technology (KL201906).

\section{Conflicts of interest/Competing interests}

Not applicable

\section{Availability of data and material}

Data and materials available

\section{Code availability}

Not applicable

\section{References}

1. Bialik, E., Stenqvist, B., Fang, Y., Östlund, Å., Furó, I., Lindman, B., Bernin, D. (2016). Ionization of cellobiose in aqueous alkali and the mechanism of cellulose dissolution. The Journal of Physical Chemistry Letters, 7(24), 5044-5048. 
2. Bian, J., Peng, F., Peng, X.-P., Peng, P., Xu, F., \& Sun, R.-C. (2012). Acetic acid enhanced purification of crude cellulose from sugarcane bagasse: structural and morphological characterization. BioResources, 7(4), 4626-4639.

3. Biermann, O., Hädicke, E., Koltzenburg, S., \& Müller-Plathe, F. (2001). Hydrophilicity and lipophilicity of cellulose crystal surfaces. Angewandte Chemie International Edition, 40(20), 3822-3825.

4. Chandrakant, P., \& Bisaria, V. S. (1998). Simultaneous bioconversion of cellulose and hemicellulose to ethanol. Crit Rev Biotechnol, 18(4), 295-331.

5. Dai L., Wang Y., Zou X., Chen Z., Liu H., Ni Y. (2020). Ultrasensitive physical, bio, and chemical sensors derived from 1-, 2-, and 3-d nanocellulosic materials. Small, 16(13), e1906567.

6. Da Silva Perez, D., Montanari, S., \& Vignon, M. R. (2003). TEMPO-mediated oxidation of cellulose III. Biomacromolecules, 4(5), 1417-1425.

7. Das K., Ray D., Bandyopadhyay NR., Sengupta S. (2010). Study of the Properties of Microcrystalline Cellulose Particles from Different Renewable Resources by XRD, FTIR, Nanoindentation, TGA and SEM. Journal of Polymers and the Environment, 18, 355-363.

8. Di Flavio, J.-L., Pelton, R., Leduc, M., Champ, S., Essig, M., \& Frechen, T. (2006). The role of mild TEMPO $-\mathrm{NaBr}-\mathrm{NaClO}$ oxidation on the wet adhesion of regenerated cellulose membranes with polyvinylamine. Cellulose, 14(3), 257-268.

9. Fu, X., Ji, H., Wang, B., Zhu, W., Pang, Z., \& Dong, C. (2020). Preparation of thermally stable and surface-functionalized cellulose nanocrystals by a fully recyclable organic acid and ionic liquid mediated technique under mild conditions. Cellulose, 27, 1289-1299.

10. Hao, J., Xu, S., Xu, N., Li, D., Linhardt, R. J., \& Zhang, Z. (2017). Impact of degree of oxidation on the physicochemical properties of microcrystalline cellulose. Carbohydr Polym, 155, 483-490.

11. Heinze, T., \& Koschella, A. (2005). Solvents applied in the field of cellulose chemistry: a mini review. Polímeros, 15(2), 84-90.

12. Heinze, T., Schwikal, K., \& Barthel, S. (2005). Ionic liquids as reaction medium in cellulose functionalization. Macromolecular bioscience, 5(6), 520-525.

13. Hirota, M., Tamura, N., Saito, T., \& Isogai, A. (2009). Oxidation of regenerated cellulose with $\mathrm{NaClO}_{2}$ catalyzed by TEMPO and NaClO under acid-neutral conditions. Carbohydrate Polymers, 78(2), 330335.

14. Hrmova, M., De Gori, R., Smith, B. J., Fairweather, J. K., Driguez, H., Varghese, J. N., \& Fincher, G. B. (2002). Structural basis for broad substrate specificity in higher plant beta-D-glucan glucohydrolases. Plant Cell, 14(5), 1033-1052.

15. Isik, M., Sardon, H., \& Mecerreyes, D. (2014). Ionic liquids and cellulose: dissolution, chemical modification and preparation of new cellulosic materials. Int J Mol Sci, 15(7), 11922-11940.

16. Isogai, A., Hänninen, T., Fujisawa, S., \& Saito, T. (2018). Review: Catalytic oxidation of cellulose with nitroxyl radicals under aqueous conditions. Progress in Polymer Science, 86, 122-148. 
17. Isogai, A., Saito, T., \& Fukuzumi, H. (2011). TEMPO-oxidized cellulose nanofibers. Nanoscale, 3(1), 7185.

18. Isogai, T., Yanagisawa, M., \& Isogai, A. (2008). Degrees of polymerization (DP) and DP distribution of cellouronic acids prepared from alkali-treated celluloses and ball-milled native celluloses by TEMPOmediated oxidation. Cellulose, 16(1), 117-127.

19. Isogai A., Kato Y. (1998). Preparation of polyuronic acid from cellulose by TEMPO-mediated oxidation. Cellulose, 5, 153-164.

20. Lasseuguette., E., Roux., D. \& Nishiyama Y. (2008). Rheological properties of microfibrillar suspension of TEMPO-oxidized pulp. Cellulose 15, 425-433.

21. Lei Dai, Yan Wang, Xuejun Zou, Zhirong Chen, Hong Liu, Yonghao Ni (2020). Ultrasensitive Physical, Bio, and Chemical Sensors Derived from 1-, 2-, and 3-D Nanocellulosic Materials. Small, 16(13), 1906567.

22. Lindman, B., Karlström, G., \& Stigsson, L. (2010). On the mechanism of dissolution of cellulose. Journal of Molecular Liquids, 156(1), 76-81.

23. Medronho, B., \& Lindman, B. (2014). Competing forces during cellulose dissolution: From solvents to mechanisms. Current Opinion in Colloid \& Interface Science, 19(1), 32-40.

24. Muhammad N., Man Z., Azmi Bustam Khalil M., Tan IM., Maitra S. (2010). Studies on the thermal degradation behavior of ionic liquid regenerated cellulose. Waste and Biomass Valorization, 1, 315321.

25. Nomura S., Kugo Y., Erata T. (2020). 13C NMR and XRD studies on the enhancement of cellulose II crystallinity with low concentration $\mathrm{NaOH}$ post-treatments. Cellulose, 27, 3553-3563.

26. Okita, Y., Fujisawa, S., Saito, T., \& Isogai, A. (2011). TEMPO-oxidized cellulose nanofibrils dispersed in organic solvents. Biomacromolecules, 12(2), 518-522.

27. Pang Z., Wang P., Dong C. (2018). Ultrasonic pretreatment of cellulose in ionic liquid for efficient preparation of cellulose nanocrystals. Cellulose, 25, 7053-7064.

28. Peng, B., Dhar, N., Liu, H., \& Tam, K. C. (2011). Chemistry and applications of nanocrystalline cellulose and its derivatives: A nanotechnology perspective. The Canadian Journal of Chemical Engineering, 89(5), 1191-1206.

29. Saito, T., Hirota, M., Tamura, N., \& Isogai, A. (2010). Oxidation of bleached wood pulp by TEMPO/ $\mathrm{NaClO} / \mathrm{NaClO}_{2}$ system: effect of the oxidation conditions on carboxylate content and degree of polymerization. Journal of Wood Science, 56(3), 227-232.

30. Saito T., Hirota M., Tamura N., Kimura S., Fukuzumi H., Heux L., Isogai A. (2009). Individualization of nano-sized plant cellulose fibrils by direct surface carboxylation using TEMPO catalyst under neutral conditions. Biomacromolecules, 10, 1992-1996.

31. Saito, T., \& Isogai, A. (2004). TEMPO-mediated oxidation of native cellulose. The effect of oxidation conditions on chemical and crystal structures of the water-insoluble fractions. Biomacromolecules, 5(5), 1983-1989. 
32. Salmén L., Bergström E. (2009). Cellulose structural arrangement in relation to spectral changes in tensile loading FTIR. Cellulose, 16, 975-982.

33. Sang, X., Qin, C., Tong, Z., Kong, S., Jia, Z., Wan, G., \& Liu, X. (2017). Mechanism and kinetics studies of carboxyl group formation on the surface of cellulose fiber in a TEMPO-mediated system. Cellulose, 24(6), 2415-2425.

34. Segal, L., Creely, J.J., Martin, A.E., Conrad, C.M. (1959). An empirical method for estimating the degree of crystallinity of native cellulose using the X-ray diffractometer. Text. Res. J. 29:786-794.

35. Tang, Z., Li, W., Lin, X., Xiao, H., Miao, Q., Huang, L., Wu, H. (2017). TEMPO-Oxidized cellulose with high degree of oxidation. Polymers, 9(9), 421-430.

36. Tahiri C., Vignon MR. (2000). TEMPO-oxidation of cellulose: Synthesis and characterisation of polyglucuronans. Cellulose, 7, 77-188.

37. Wang, S., Lu, A., \& Zhang, L. (2016). Recent advances in regenerated cellulose materials. Progress in Polymer Science, 53, 169-206.

38. Yang H, Yan R., Chen H., Lee DH., Zheng C (2007). Characteristics of hemicellulose, cellulose and lignin pyrolysis. Fuel, 86, 1781-1788.

39. Yang Y-J., Shin J-M., Kang TH., Kimura S., Wada M., Kim U-J (2014). Cellulose dissolution in aqueous lithium bromide solutions. Cellulose, 21, 1175-1181.

40. Yoo, C. G., Zhang, S., \& Pan, X. (2017). Effective conversion of biomass into bromomethylfurfural, furfural, and depolymerized lignin in lithium bromide molten salt hydrate of a biphasic system. RSC Advances, 7(1), 300-308.

\section{Figures}




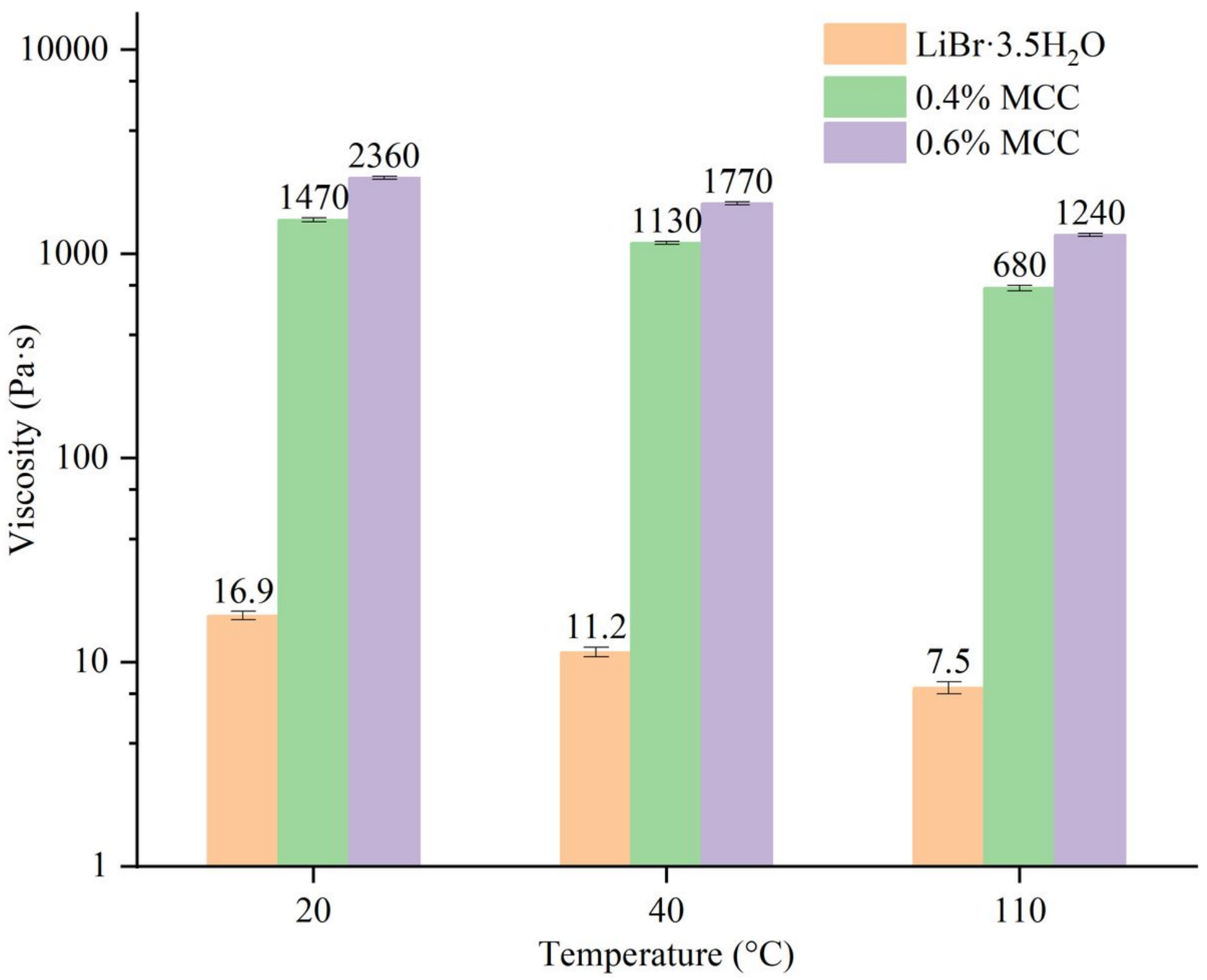

Figure 1

Viscosity of $\mathrm{LiBr} \cdot 3.5 \mathrm{H} 2 \mathrm{O}$ at varying temperature and $\mathrm{MCC}$ concentrations 

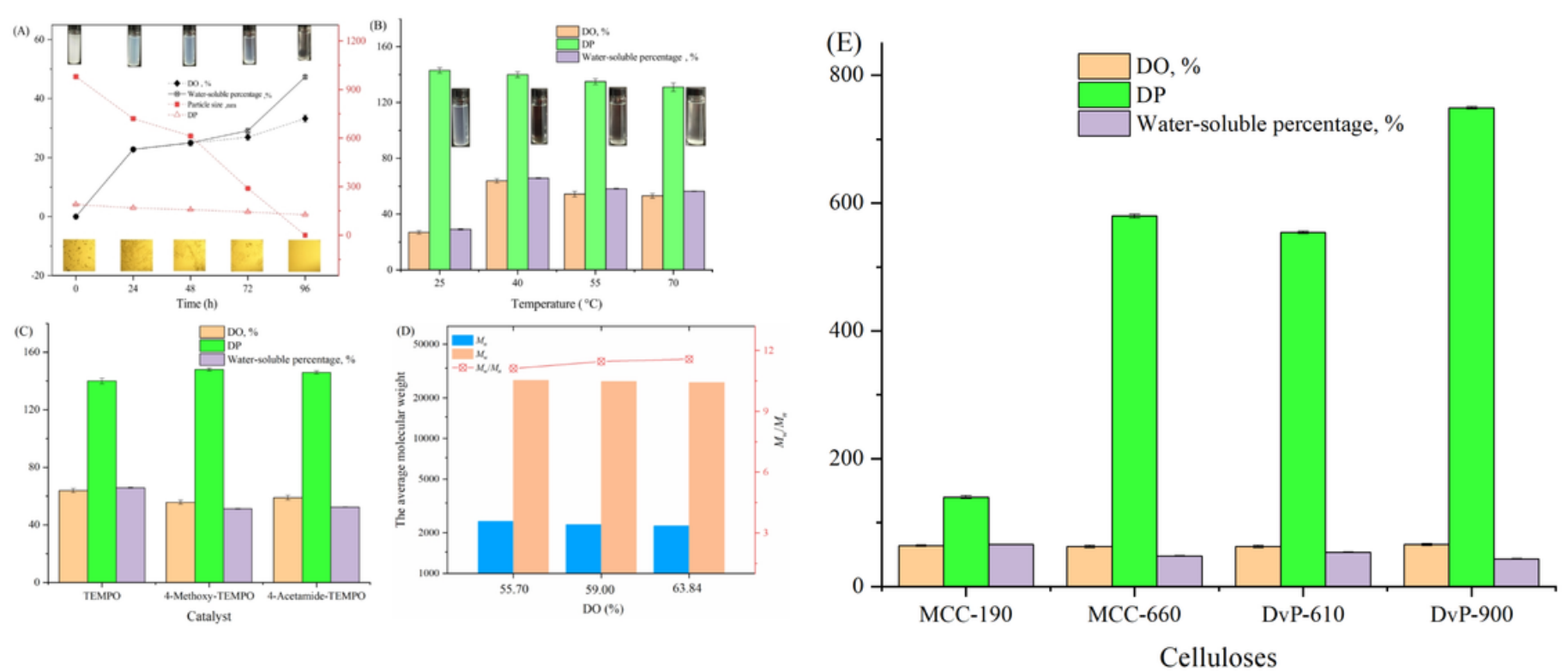

Figure 2

Effect of reaction conditions on oxidation of cellulose, (A) DO, particle size and appearances of oxidized cellulose with varying reaction time, (B) DO and appearances of oxidized cellulose with varying temperature, and effect catalyst on DO (C) and the molecular weight (D) of the oxidized cellulose, and (E) effect celluloses on DO. 


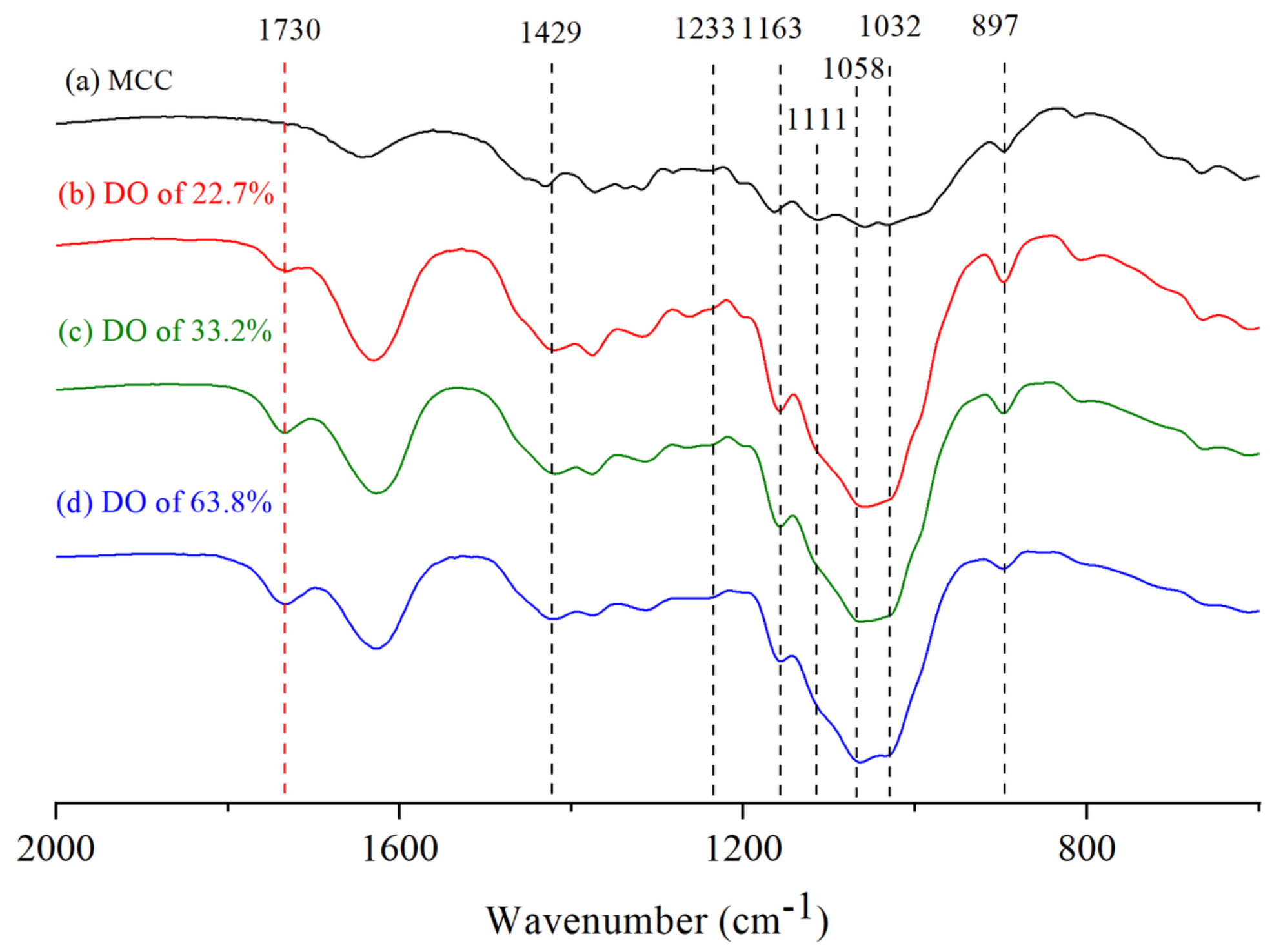

Figure 3

FTIR spectrum of MCC and the oxidized celluloses with varying DO 


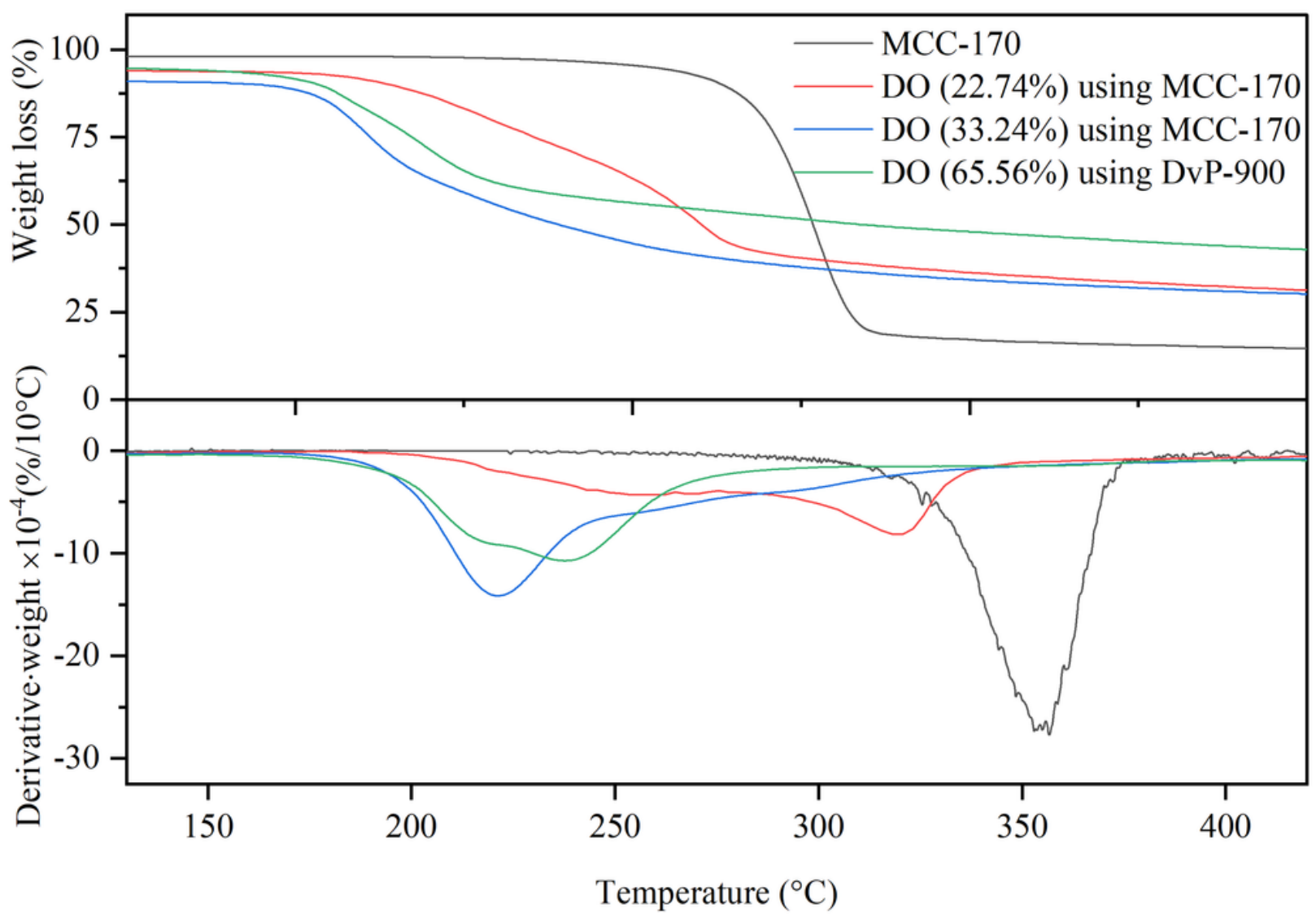

Figure 4

The TGA curves of MCC and the oxidized celluloses with different DO 


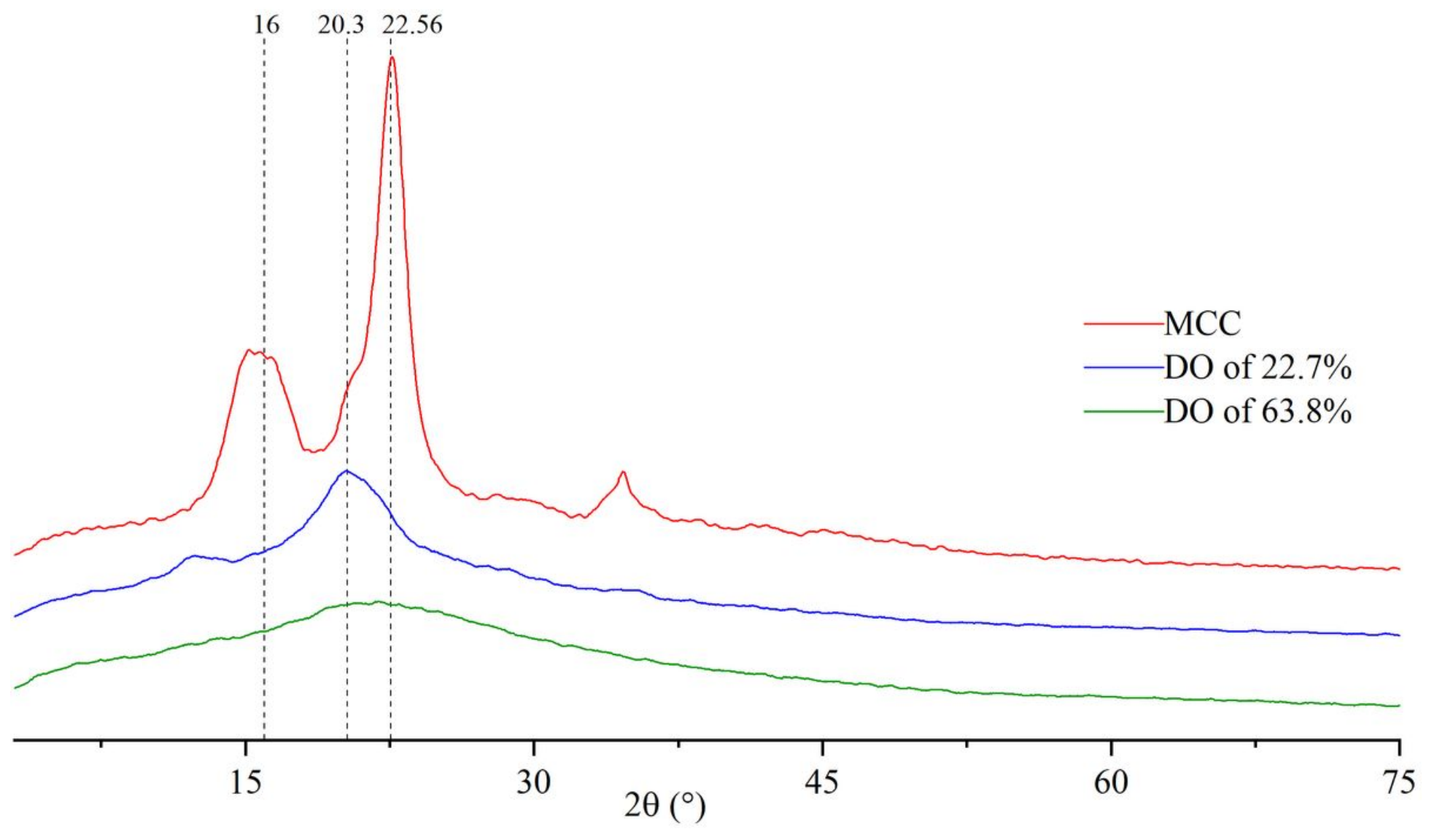

Figure 5

XRD spectra of MCC and the oxidized celluloses with different DO 


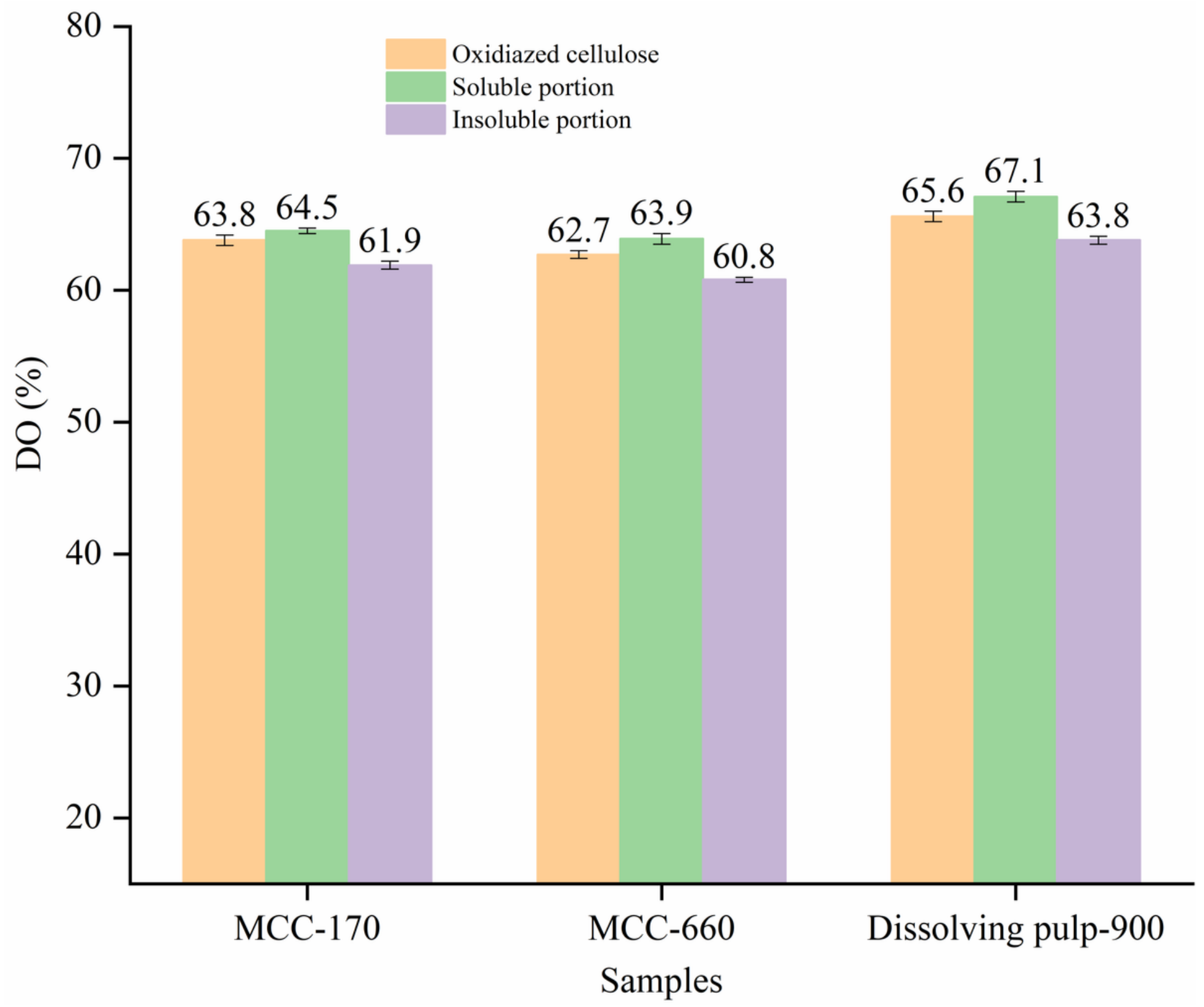

Figure 6

DO of the oxidized celluloses and its soluble and insoluble portions after fractionation 

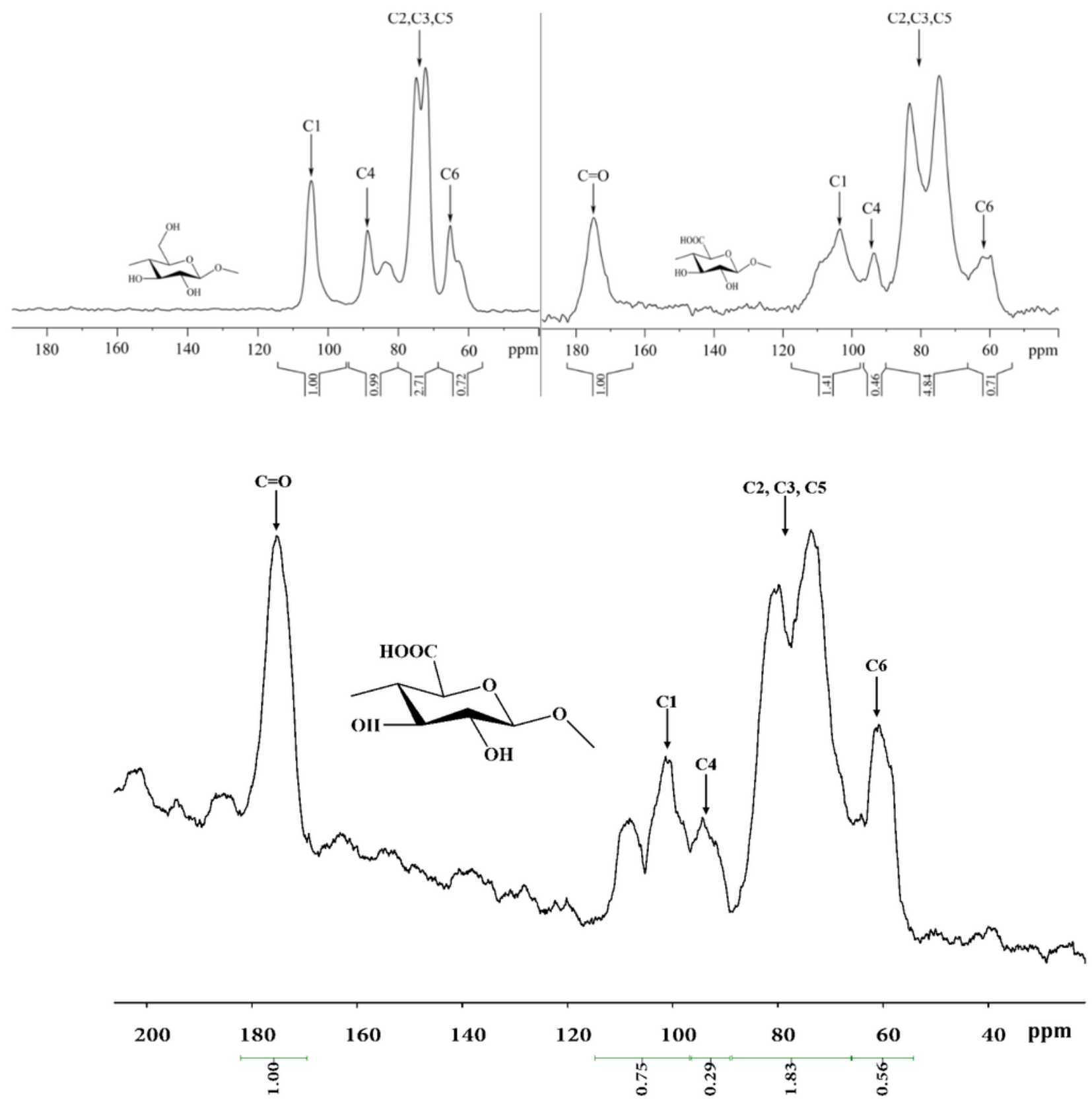

Figure 7

13C NMR spectra of MCC, oxidized cellulose and water-soluble oxidized cellulose

\section{Supplementary Files}

This is a list of supplementary files associated with this preprint. Click to download.

- 0448FAD31BB6C9D52B8395019F8C5A1C.jpg 
- Supplementarymaterial.docx

Page 23/23 